\title{
A diffusion approximation based on renewal processes with applications to strongly biased run-tumble motion
}

\section{Thygesen, Uffe Høgsbro}

\section{Published in:}

Bulletin of Mathematical Biology

Link to article, DOI:

10.1007/s11538-016-0155-3

Publication date:

2016

Document Version

Peer reviewed version

Link back to DTU Orbit

Citation (APA):

Thygesen, U. H. (2016). A diffusion approximation based on renewal processes with applications to strongly biased run-tumble motion. Bulletin of Mathematical Biology, 78(3), 556-579. https://doi.org/10.1007/s11538-0160155-3

\section{General rights}

Copyright and moral rights for the publications made accessible in the public portal are retained by the authors and/or other copyright owners and it is a condition of accessing publications that users recognise and abide by the legal requirements associated with these rights.

- Users may download and print one copy of any publication from the public portal for the purpose of private study or research.

- You may not further distribute the material or use it for any profit-making activity or commercial gain

- You may freely distribute the URL identifying the publication in the public portal 
Noname manuscript No.

(will be inserted by the editor)

\title{
A diffusion approximation based on renewal processes
}

\section{with applications to strongly biased run-tumble motion}

\section{Uffe Høgsbro Thygesen}

the date of receipt and acceptance should be inserted later

\begin{abstract}
We consider organisms which use a renewal strategy such as runtumble when moving in space, for example to perform chemotaxis in chemical gradients. We derive a diffusion approximation for the motion, applying a central limit theorem due to Anscombe for renewal-reward processes; this theorem has not previously been applied in this context. Our results extend previous work, which has established the mean drift but not the diffusivity. For a classical model of tumble rates applied to chemotaxis, we find that the resulting chemotactic drift saturates to the swimming velocity of the organism when the chemical gradients grow increasingly steep. The dispersal becomes anisotropic in steep gradients, with larger dispersal across the gradient than along the gradient. In contrast to one-dimensional settings, strong bias increases dispersal. We next include Brownian rotation in the model and find that, in limit of high chemotactic sensitivity, the chemotactic drift is $64 \%$ of the swimming Center for Ocean Life, National Institute of Aquatic Resources, Technical University of Denmark, Jægersborg Allé 1, DK-2920 Charlottenlund, Denmark. E-mail: uht@aqua.dtu.dk
\end{abstract}


velocity, independent of the magnitude of the Brownian rotation. We finally derive characteristic time scales of the motion that can be used to assess if the diffusion limit is justified in a given situation. The proposed technique for obtaining diffusion approximations is conceptually and computationally simple, and applicable also when statistics of the motion is obtained empirically or through Monte Carlo simulation of the motion.

Keywords Movement models, Diffusion approximation, Run-tumble strategy, Renewal process, Chemotaxis

\section{Introduction}

Chemotaxis, the ability of organisms to climb the gradient of a given chemical and thus reach regions with elevated concentrations of that chemical, has been investigated empirically and theoretically for more than a century (Adler, 1966; Keller and Segel, 1971; Alt, 1980; Okubo and Levin, 2001; Colin et al, 2014; Xue, 2015). Chemotaxis is ubiquitous in biology (Kay et al, 2008), occurring for example in bacterial infections and their immune response, formation of biofilms, spread of cancer, and bacterial colonization of marine snow. In a heterogeneous environment, chemotactic abilities determine the nutrient uptake of the organism, and a quantitative understanding of the phenomenon is necessary for assessing ecological rates and aggregations of organisms.

For bacteria, the run-tumble model (Brown and Berg, 1974; Berg, 1993) is particularly well studied. In this model, bacteria swim with constant velocity until they tumble and reorient. The tumble rate is increased when the 
bacterium experiences decreasing concentrations, and the net effect is a chemotactic drift in the direction of the gradient (see details in section 3). At the same time, the randomness in the behavior implies a dispersal of individuals. This model leads directly to stochastic simulation model of the individual bacterium, e.g. (Jackson, 1989), which can assess the performance of the chemotactic strategy. Even if this model does not capture all important dynamics of specific bacteria such as E. coli (Cluzel et al, 2000; Xue, 2015), the run-tumble model of chemotaxis is an archetypal example of a search strategy employed by organisms to identify favorable regions in space. Here, we examine the analytical properties of this model, in particular in the situation of strong bias which corresponds to a bacterium experiencing steep gradients.

When studying run-tumble motion and other search strategies, the issue of scales often arises because the single run can be considered short relative to the scales of environmental heterogeneity, e.g. a chemical gradient. Such questions of scale are ubiquitous in mathematical biology (Hastings et al, 2011; Benhamou, 2014), with different models being appropriate to describe a given phenomenon at different scales. From a mathematical point of view, this raises the question of consistency across scales: Are two different models, each conceived to represent movement at a particular scale, consistent in the sense that they give identical predictions at some scale or in some limit? In many cases, movement may be represented on large scales by an advection-diffusion equation (Okubo and Levin, 2001), corresponding to the organism performing a biased random walk. In this coarse-grained model, the details of the 
individual run, and possibly other internal dynamics (Grünbaum, 2000; Xue, 2015), is summarized in a drift term giving the mean velocity, and a diffusivity parametrizing the dispersal.

The now classical approach to obtaining such diffusion approaches (Alt, 1980) builds on the seminal work by Patlak (1953). This analysis applies singular perturbation analysis to the evolution equation, or Master equation, governing the probability distribution of the state of the bacteria. A quite general result was obtained by Othmer and Hillen (2002). Studies applying this approach include Grünbaum (1999), who considered foragers searching for discrete patches and emphasized the connection between observable statistics of motion and the diffusion approximation, Bearon and Pedley (2000) who included the rotation due to shear flow, aiming to describe the performance of the bacteria in a turbulent environment, and Xue (2015) who emphasized the intracellular biochemistry.

In the interest of technical simplicity, many applications follow Keller and Segel (1971) and consider only the first term in the expansion which is a linear relationship between chemical gradient and the resulting chemotactic drift. Similarly, to first order, the diffusivity is independent of the gradient. This, however, does not always lead to useful results, since it predicts that the chemotactic drift may exceed the actual swimming speed of the organism, if the chemical gradient is steep enough. This situation arises, for example, for bacteria attracted to marine snow, where thin boundary layers may form which feature steep chemical gradients (Kiørboe et al, 2001). In general, strong 
patchiness is a common condition in aquatic ecology and in biology in general, and therefore the response to strong cues is important for the fitness of organisms and the emergent ecosystem properties. This justifies not only empirical studies of behavior in strong gradients, but also a detailed analysis of models of behavior in strong gradients.

In a one-dimensional setting, the model can be cast as the telegraph equation, for which a sigmoid relation was found (Rivero et al, 1989) between the gradient of the chemoattractant and the resulting chemotactic drift. For the diffusivity, this study concluded that strong gradients reduced the diffusivity: In the limit of infinitely strong gradients, cells would all move up gradient with constant velocity, ultimately yielding a diffusivity of 0. Bearon (2003) used the framework of generalized Taylor dispersion (Frankel and Brenner, 1989) and considered non-linear effects in a three dimensional setting. In this study, the first terms in an expansion of the diffusivity also showed that that strong gradients reduced the diffusivity in the direction of the gradients.

The technical challenges of singular perturbation analysis make it useful to consider alternative ways to derive diffusion limits, of which one is the theory of stochastic processes. One approach is to derive the diffusivity from mean-square considerations (Sandoval et al, 2014), essentially integrating the autocorrelation function of the velocity process. Another approach is to divide the motion into independent segments. This was pursued by Bearon and Pedley (2000) who showed that chemotactic drift could be determined equally well with a perturbation expansion and with the law of large numbers. In this paper 
we continue in this direction, and assume that the motion can be divided into stochastically independent segments, i.e. runs. This allows us to apply the machinery of stochastic processes of renewal-reward type, in particular a central limit theorem due to Anscombe (1952) which appears to be practically unused in mathematical biology in general and in particular in the study of random motion of organisms. A similar approach was taken by Bearon (2001); the end result relates statistics of the single run to coarse-grained statistics of the motion, i.e. the drift and diffusivity.

When making use of diffusion approximations, it is necessary to assess if the separation of scales is justified. To this end, we investigate the time scale of velocity fluctuations, and compare two measures: The average time since last tumble, and the decorrelation time of the velocity, i.e. the Lagrangian time scale. As we shall see, these two measures both indicate that velocity fluctuations are more persistent in steeper gradients. This also opens for the possibility that processes which may be ignored in weak gradients can be decisive in strong gradients. As an example of this, we examine the effect of Brownian rotation with strong chemotactic sensitivity.

The main advantage of our approach, compared for example to singular perturbation analysis, is technical simplicity. While the derivation of limit theorems for renewal processes and other stochastic processes may be considered a specialized discipline, the application of such theorems requires only straightforward analysis to yield the necessary statistics of the microscale model. In the examples in the following, we employ simple mathematical analysis, Monte 
Carlo simulation, and standard numerical analysis of diffusion equations, to this end. Once such microscale statistics have been obtained, elementary calculations yield the coarse-grained statistics. The technical simplicity of this analysis provides an opportunity to address non-trivial questions, e.g. if strong bias reduces or increases diffusivity, and how large spatial scales are required for a diffusion approximation to be justified.

The paper is organized as follows: In section 2 we establish the framework of using renewal processes to describe run-tumble motion such as chemotaxis, and apply Anscombe's theorem to obtain a diffusion limit of such motion. In section 3 we go through the detailed calculations when applying the result to the classical run-tumble model. In section 4 we consider timescales which characterize the motion and which can be used to assess if a diffusion approximation is reasonable. In section 5 we consider the effect of Brownian rotation. Finally section 6 offers a discussion and some conclusions.

\section{Diffusion approximations based on renewal-type velocity} processes

In this section we consider movement patterns which can be divided into stochastically independent steps. This is a natural first description of the runtumble motion of bacteria and other organisms, in that the runs are assumed to be independent.

First some notation: We use $t_{i}$ to denote the duration of the $i$ th run, and $x_{i}$ the displacement during the same run. Our focus is the three-dimensional 
case $x_{i} \in \mathbf{R}^{3}$, but the results in this section apply regardless of the number of dimensions. $T_{i}$ is the time when the $i$ th runs starts; we refer to this as the $i$ th tumble. $X_{i}$ is the position at time $T_{i}$. Hence

$$
t_{i}=T_{i+1}-T_{i}
$$

and

$$
x_{i}=X_{i+1}-X_{i} \quad .
$$

We do not consider positions or velocities during the runs themselves, only at tumbles. We therefore introduce the position at last tumble

$$
\bar{X}_{t}=X_{N(t)}
$$

where $N(t)=\max \left\{n \geq 0: T_{n} \leq t\right\}$ is the counting process, giving the number of tumbles before time $t \geq 0$. Notice that the process $\bar{X}_{t}$ is a position jump process or what is termed a kangaroo process by Othmer et al (1988). The objective of the following is to approximate the distribution of $\bar{X}_{t}$ with a Gaussian when $t$ is large, and thus reach a diffusion approximation of the motion.

We now make the crucial assumption that runs $i$ and $j$ are stochastically independent for $i \neq j$, and that the duration and displacement of run $i$ have a known distribution which is independent of $i$ and has finite first and second order statistics:

$$
\mathbf{E}\left(\begin{array}{c}
t_{i} \\
x_{i}
\end{array}\right)=\left(\begin{array}{c}
\tau \\
\mu
\end{array}\right), \quad \mathbf{V}\left(\begin{array}{c}
t_{i} \\
x_{i}
\end{array}\right)=\left[\begin{array}{cc}
\sigma_{t}^{2} & \rho^{\prime} \\
\rho & \Sigma_{x}
\end{array}\right] .
$$


To analyse the long-term behaviour of the process $\bar{X}_{t}$, we may apply the law of large numbers. The mean velocity during the first $n$ steps is $\bar{v}_{n}=X_{n} / T_{n}$ which approaches $\mu / \tau$ as $n \rightarrow \infty$, almost surely. This follows directly from the independence of runs, using that $n^{-1} \sum_{i=1}^{n} x_{i} \rightarrow \mu$ and $n^{-1} \sum_{i=1}^{n} t_{i} \rightarrow \tau$ almost surely, as $n \rightarrow \infty$. In the context of bacterial chemotaxis, this result for the mean velocity is used by Bearon and Pedley (2000); Locsei (2007).

To study the behavior after a long time, i.e. as $t \rightarrow \infty$, rather than after many runs, i.e. as $n \rightarrow \infty$, it is useful to note that the tumble process $\left\{T_{i}\right\}$ is a point process on $\mathbf{R}$ of renewal type (Grimmett and Stirzaker, 1992); the defining property being exactly that times $\left\{t_{i}\right\}$ between tumbles are independent and identically distributed. The combined process $\left(T_{i}, x_{i}\right)$ is thus a renewal-reward process, where the displacement $x_{i}$ during a run is a reward associated with the renewal point $T_{i}$. With this terminology, the position $X_{n}$ after a run is the cumulated reward. Asymptotic properties of renewal processes is a well-studied subject in stochastic processes. The renewal-reward theorem states that $\bar{X}_{t} / t \rightarrow \mu / \tau$ as $t \rightarrow \infty$, almost surely; i.e. the "long time limit" agrees with the "many jumps limit". The elementary renewal theorem for renewal-reward processes states that also $t^{-1} \mathbf{E} \bar{X}_{t} \rightarrow \mu / \tau$ as $t \rightarrow \infty$, i.e. the ensemble mean (corresponding to averaging over many bacteria moving independently) agrees with the long-time average for a single arbitrarily chosen bacterium. To obtain a similar expression for the variance, we employ the following central limit theorem due to Anscombe (1952), see also Grimmett and Stirzaker (1992): 
Theorem 1 (Anscombe) Let $z_{i}$ be independent identically distributed scalar random variables with mean 0 and variance $\sigma^{2}$. Let $S_{n}=\sum_{i=1}^{n} z_{i}$, and let $N_{t}$ be an integer-valued process such that $t^{-1} N(t) \rightarrow 1 / \tau>0$ in probability as $t \rightarrow \infty$. Then

$$
\frac{S_{N(t)}}{\sqrt{t / \tau}} \rightarrow N\left(0, \sigma^{2}\right)
$$

in distribution as $t \rightarrow \infty$.

Background information, such as definitions of convergence in probability and in distribution, can be found in Grimmett and Stirzaker (1992). With this theorem, we obtain the following result for the asymptotic mean and variance of the position in the run-tumble motion:

Proposition 1 In the renewal-reward model of the run-tumble motion, the displacement $\bar{X}_{t}$ is asymptotically normal distributed:

$$
\frac{1}{\sqrt{t}}\left(\bar{X}_{t}-t \frac{\mu}{\tau}\right) \rightarrow N(0, \Sigma / \tau)
$$

in distribution as $t \rightarrow \infty$, where

$$
\Sigma=\Sigma_{x}+\frac{\mu \mu^{\prime}}{\tau^{2}} \sigma_{t}^{2}-\frac{1}{\tau}\left(\mu \rho^{\prime}+\rho \mu^{\prime}\right)
$$

The proof is given in appendix A. A similar result is derived by Bearon (2001, section 3.3) for the "many steps limit" without using Anscombe's theorem; see in particular equations (3.30), (3.34) and (3.37) therein.

Notice that asymptotically, both the mean displacement $\mathbf{E} \bar{X}_{t} \approx t \mu / \tau$ and the variance $\mathbf{V} \bar{X}_{t} \approx t \Sigma / \tau$ scale linearly with time, as expected and in agreement with standard diffusion theory. Notice also that in the unbiased case 
$\mu=0$, we recover the standard expression $\Sigma=\Sigma_{x}$ which can be obtained with elementary arguments. The correction $\Sigma-\Sigma_{x}$ is of second order in the bias $\mu$-assuming that the covariance $\rho$ is of first order which will typically be the case. As a trivial example where the correction term matters, consider the case where the velocity is fixed and common to all runs, i.e. $x_{i}=v \cdot t_{i}$ for fixed velocity vector $v \in \mathbf{R}^{n}$, while the run lengths $t_{i}$ have an arbitrary distribution

with mean $\tau$ and variance $\sigma_{t}^{2}$. For this example we find $\mu=v \cdot \tau, \Sigma_{x}=v v^{\prime} \cdot \sigma_{t}^{2}$, and $\rho=v \cdot \sigma_{t}^{2}$, yielding the obvious result $\Sigma=0$.

The theorem allows to approximate the motion, when the time $t$ is large, with a Wiener process (Brownian motion) with drift; i.e. a biased random walk in continuous time and space. Here, the drift is $\mu / \tau$ and the diffusivity is $\Sigma / 2 \tau$.

\section{Diffusion limit of a standard model of run-tumble chemotaxis}

While the preceding section was for a general model of motion which can be divided into independent runs, we now turn to the standard run-tumble model (Brown and Berg, 1974; Jackson, 1989) of bacterial chemotaxis, aiming to obtain explicit expressions for chemotactic drift and dispersal. We consider a bacterium in still water which experiences a time-invariant concentration of the chemoattractant. The bacterium has position $X_{t} \in \mathbf{R}^{3}$ and velocity $V_{t}=d X_{t} / d t \in \mathbf{R}^{3}$. Runs, i.e. periods of constant velocity, are separated by instantaneous tumbles at which point a new velocity is sampled uniformly from the sphere $\{v:|v|=U\}$. We assume that tumbles arrive randomly at 
a rate $\lambda\left(X_{t}, V_{t}\right)$ so that $\left(X_{t}, V_{t}\right)$ together form a Markov process. We note that this assumption implies that we ignore intracellular state dynamics, an assumption that will not always be justified.

The classical model (Brown and Berg, 1974) is as follows: The bacterium measures the concentration of the chemoattractant by monitoring the fraction of bound chemoreceptors at its surface. This fraction, $P\left(X_{t}\right)$, depends on the local concentration $C$ of the chemical attractant:

$$
P(x)=\frac{C(x)}{K+C(x)}
$$

where $x \in \mathbf{R}^{3}$ and $K$ is a constant parameter, the half-saturation concentration. Here we have assumed steady-state in the receptor dynamics, see also Jackson (1987, 1989); Kiørboe and Jackson (2001); Locsei (2007), an assumption that may not be valid, in particular when the cell experiences rapidly changing chemical concentrations Xue (2015).

Tumbles are stimulated when the fraction $P\left(X_{t}\right)$ decreases with time, and suppressed when $P\left(X_{t}\right)$ increases. Specifically, the tumble rate $\lambda$ can be computed from the rate of change in $P\left(X_{t}\right)$ according to the formula

$$
\log \lambda\left(X_{t}, V_{t}\right)=\log \lambda_{0}-\alpha \frac{d}{d t} P\left(X_{t}\right)
$$

where $\lambda_{0}$ and $\alpha$ are constant parameters. In summary

$$
\lambda(x, v)=\lambda_{0} \cdot \exp \left(-\alpha \frac{K}{(K+C)^{2}} v \cdot \nabla C\right)
$$

where $C$ and $\nabla C$ are evaluated at $x \in \mathbf{R}^{3}$. 
The customary approach is at this point to summarize the model in terms of a Master equation, which governs the temporal evolution of the probability distribution of the state (position and velocity). Next, to assume a separation of scales which allows a singular perturbation analysis of this equation, resulting in a diffusion limit. In contrast, the approach in the following is to assume a separation of scales which allows us to consider the run statistics constant over several subsequent runs, and thus apply the central limit theorem of the previous section. We therefore introduce the dimensionless quantity $\beta$

$$
\beta(x)=\alpha \frac{K}{(K+C(x))^{2}}|\nabla C(x)| U
$$

$\beta$ combines chemotactive sensitivity and strength of the chemical gradient, so that the absolute value of $\beta$ indicates the strength of the chemotaxis and thus the bias in the resulting motion. For the homogeneous case where $\beta(x)$ is a constant function of $x \in \mathbf{R}^{3}$, it is convenient to introduce a coordinate system such that the $z$-axis points in the direction of the chemical gradient. With this, we obtain the following:

Theorem 2 Assume that the tumble rate $\lambda(x, v)$ is independent of $x \in \mathbf{R}^{3}$ and has the form

$$
\lambda(x, v)=\lambda_{0} \exp \left(-\beta \frac{v \cdot e_{z}}{U}\right)
$$

where $e_{z}$ is the unit vector in the direction of the $z$-axis. Then the diffusion limit of the run-tumble process is given by

$$
\frac{1}{\sqrt{t}}\left(X_{t}-X_{0}-v t\right) \rightarrow N(0,2 D) \text { in distribution as } t \rightarrow \infty
$$


where the drift is $v=U \cdot\left(0,0, v_{z}\right)$ with

$$
v_{z}=\left(\operatorname{coth} \beta-\frac{1}{\beta}\right)
$$

while the diffusivity is $D=\operatorname{diag}\left(D_{x}, D_{y}, D_{z}\right) \cdot U^{2} / \lambda_{0}$ with

$$
D_{x}=D_{y}=\frac{2 \beta \cosh 2 \beta-\sinh 2 \beta}{8 \beta^{2} \sinh \beta} \text { and } D_{z}=\frac{1}{4} \frac{\sinh 2 \beta-2 \beta}{\beta^{2} \sinh \beta} \text {. }
$$

The theorem applies when the tumble rate $\beta$ is constant, but in many applications $\beta$ will depend on space, as for example in the model (1). We return to this issue in the following section.

The proof of the theorem consists of computing the statistics of a single run of the motion and apply proposition 1 . These statistics are obtained with fairly elementary integrals over space of possible velocities, i.e. the surface of the sphere. The details are found in appendix B. We note that the same results could have been found inserting the specific form (2) of the tumble rate into the general formulas of Bearon (2001, section 4.1); the actual computations being parallel despite differences in the framing.

The results of the theorem are shown graphically in figure 1 . The figure also includes results from a Monte Carlo simulation which was performed to verify the results; see details in appendix $\mathrm{C}$. When $\beta \rightarrow 0$, the following approximations are useful

$$
\begin{gathered}
v_{z}=\frac{1}{3} \beta-\frac{1}{45} \beta^{3}+o\left(\beta^{4}\right) \\
D_{x}=D_{y}=\frac{1}{3}+\frac{7}{90} \beta^{2}+o\left(\beta^{3}\right)
\end{gathered}
$$



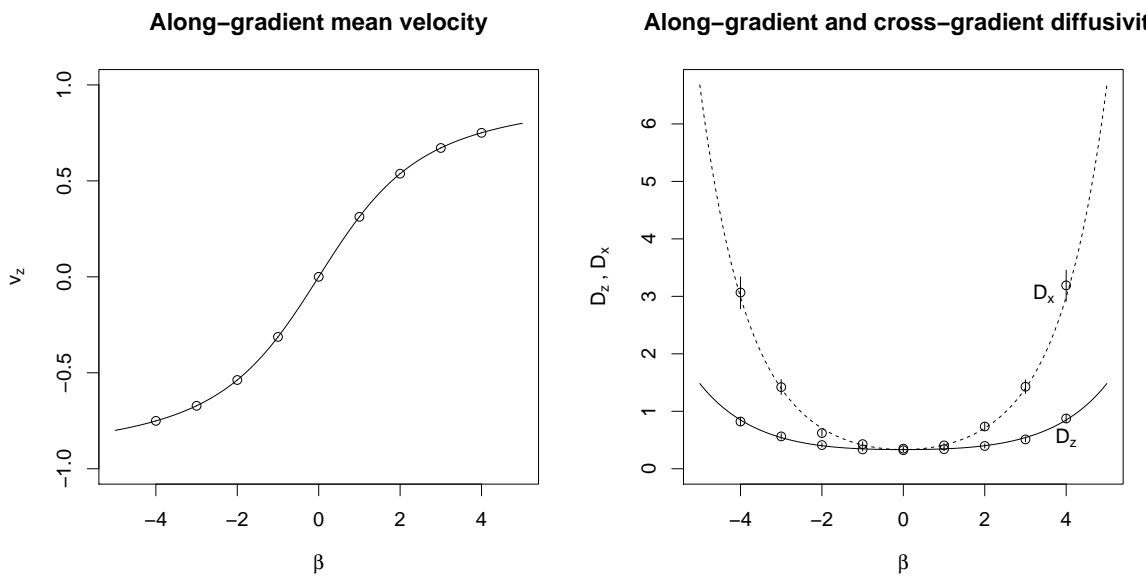

Fig. 1 The dimensionless chemotactic velocity and diffusivity, obtained with the central limit theorem, as function of the dimensionless steepness parameter $\beta$. Positive $\beta$ corresponds to an attractant while negative $\beta$ corresponds to a repellent. Circles show estimates obtained with a Monte Carlo simulation of the run-tumble model (see details in appendix C) while vertical bars display the associated $95 \%$ confidence intervals; this simulation verifies the theoretical results.

$$
D_{z}=\frac{1}{3}+\frac{1}{90} \beta^{2}+o\left(\beta^{3}\right)
$$

Note that the diffusivity $D$ contains anisotropic terms of second order in $\beta$, In turn, in steep gradients $\beta \rightarrow \infty$ an elementary analysis yields the following approximations:

$$
\begin{aligned}
v_{z} & =1-\frac{1}{\beta}+O\left(e^{-\beta}\right) \\
D_{x} & =\frac{e^{\beta}}{4 \beta}(1+O(1 / \beta)) \\
D_{z} & =\frac{e^{\beta}}{4 \beta^{2}}\left(1+O\left(\beta e^{-2 \beta}\right)\right)
\end{aligned}
$$


Note that the diffusivity is enhanced both in the direction of the chemical gradient (here, the $z$-axis) and in the directions perpendicular to this gradient, but mostly so in the directions perpendicular to the gradient.

\section{Time scales and the validity of the diffusion approximation}

The validity of the diffusion approximation relies on a separation of scales, in that the environment must be nearly constant during a sequence of runs, so that $\beta(x)$ can be considered locally constant and asymptotic statistics based on constant $\beta$ apply. To assess the validity of this approximation, it is useful to examine time scales which characterize the fluctuations in the velocity process and the individual run. We compute this time scale for the model with frozen parameters, i.e. as in theorem 2, and assuming stochastic steady state.

A first candidate time scale is the mean duration of a run, i.e. $\tau$. However, this will underestimate the correlation in the velocity process in the case of steep gradients: There will be many short runs going in the wrong direction which will affect the mean duration of a run, but not cause the velocity process to decorrelate. A more useful statistic is the mean duration of the run containing a given point of time, i.e. $\mathbf{E} t_{N(t)}$. The fact that this differs from the mean run duration is known as the bus stop paradox (or the inspection paradox) in the literature of stochastic processes (Grimmett and Stirzaker, 1992).

By symmetry, the duration of the run containing a typical point is related to the time since last tumble, $A_{t}=t-T_{N(t)}$, which is known as the age in renewal theory. Specifically, 


$$
\lim _{t \rightarrow \infty} \mathbf{E} t_{N(t)}=\lim _{t \rightarrow \infty} 2 \mathbf{E} A_{t}
$$

To pursue this time constant, we once again use the framework of renewal processes (Grimmett and Stirzaker, 1992). Let $G$ denote the complementary cumulative distribution function of the run duration $t_{i}$, i.e. $G(s)=\mathbf{P}\left(t_{i}>\right.$ $s)$. Let $f$ be the probability density function of the run durations, $f(s)=$ $-G^{\prime}(s)$. In stationarity, the probability density of the age $A_{t}$ is (Grimmett and Stirzaker, 1992)

$$
f_{A}(s)=\frac{1}{\tau} G(s)
$$

where, as before, $\tau=\mathbf{E} t_{i}$ is the mean run duration. It follows that the expected age is

$$
\mathbf{E} A_{t}=\frac{1}{\tau} \int_{0}^{\infty} s G(s) d s=\frac{1}{\tau} \int_{0}^{\infty} \frac{1}{2} s^{2} f(s) d s=\frac{1}{2} \frac{\mathbf{E} t_{i}^{2}}{\mathbf{E} t_{i}}=\frac{1}{2} \frac{\sigma_{t}^{2}}{\tau}+\frac{1}{2} \tau
$$

where, as before, $\sigma_{t}^{2}=\mathbf{V} t_{i}$ is the variance of the run duration. Here we have used integration by parts. For the run-tumble motion, we get

$$
\mathbf{E} A_{t}=\frac{1}{2 \lambda_{0}} \frac{\sinh 2 \beta}{\sinh \beta}
$$

Since $\mathbf{E} A_{t}=1 / \lambda_{0}$ in the unbiased case, it is convenient to use this time scale rather than the duration $\mathbf{E} t_{N(t)}$, which, as we saw, differs with a factor of two.

A final way to measure the time constant is the Lagrangian time scale $T_{L}$ which is obtained by integrating the autocorrelation function of the velocity. 


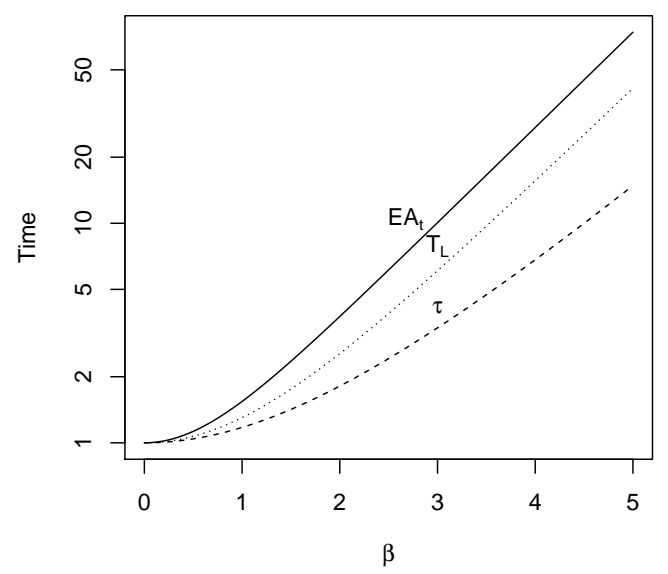

Fig. 2 Dimensionless time constants of the run-tumble model, as function of the dimensionless parameter $\beta$ describing chemotactic strength. Note logarithmic scale on the ordinate.

Equivalently (Taylor, 1921) the Lagrangian time scale is the ratio between the diffusivity and the variance of velocity fluctuations. A discussion relating to the differences between these time scales can be found in Bearon (2001, section 4.1). When the velocity process is anisotropic, as in our situation, we average over the three dimensions and obtain:

$$
T_{L}=\frac{D_{x}+D_{y}+D_{z}}{\mathbf{E}\left|V_{t}\right|^{2}-\left|\mathbf{E} V_{t}\right|^{2}}=\frac{1}{\lambda_{0}} \frac{2 \beta \sinh ^{3}(\beta)}{2 \beta \sinh (2 \beta)-\cosh (2 \beta)+1-2 \beta^{2}}
$$

The three time constants $\tau, \mathbf{E} A_{t}$, and $T_{L}$ are seen in figure 2 as function of $\beta$ when $\lambda_{0}=1$. Note that they all agree in the unbiased case $\beta=0$ where the time constant is $1 / \lambda_{0}$, and that the time constants increase with the gradient, as is to be expected. When $\beta \rightarrow \infty$ we have

$$
\mathbf{E} A_{t}=\frac{e^{\beta}}{2 \lambda_{0}}(1+o(1)), \quad T_{L}=\frac{e^{\beta}}{4 \lambda_{0}}(1+o(1)), \quad \tau=\frac{e^{\beta}}{2 \beta}(1+o(1)) .
$$


Note that the mean run duration $\tau$ is the smallest of the three time constants, and that $\mathbf{E} A_{t}$ and $T_{L}$ differ with a factor 2 when $\beta \rightarrow \infty$.

These time scales can be used to assess the validity of the diffusion approximation in the following way: The time scales $\mathbf{E} A_{t}$ and $T_{L}$ translates to characteristic length scales; e.g. $U \cdot \mathbf{E} A_{t}$ is the typical distance traveled since last tumble. For the diffusion approximation to be valid, the tumble rate $\beta$ must be constant over many subsequent runs, so that spatial fluctuations in $\beta$ over the length scale $U \cdot \mathbf{E} A_{t}$ must be negligible.

\section{Brownian rotation and strong chemotactic sensitivity}

The conclusion from the previous is that as gradient steepness tends to infinity, or the chemotactic sensitivity tends to infinity, the chemotactic velocity in the direction of the gradient approaches the swimming speed while the diffusivity tends to infinity. However, since also the time constants of the motion tend to infinity, these conclusions are not robust towards modifications or additions to the model. To illustrate this, we here examine the effect of Brownian rotation (Berg, 1993), i.e. during runs we let the velocity vector perform Brownian motion on the sphere. This will impair the organism's ability to align with the gradient and thus decrease the chemotactic velocity. At the same time, it will decrease the decorrelation time of the velocity process and therefore decrease the diffusivity.

We consider the limiting case of an infinitely steep gradient, or equivalently, infinitely high chemotactic sensitivity, i.e. we let $\beta \rightarrow \infty$ in (2). In this limit, 
the tumble rate is infinite when the direction is down-gradient $\left(V_{t} \cdot e_{z}<0\right)$, and 0 when the direction is up-gradient $V_{t} \cdot e_{z}>0$. In words, the bacterium will tumble instantaneously when it is swimming down-gradient, and tumble again as needed until it is headed up-gradient. It will never tumble while swimming up-gradient.

Thus, the velocity $V_{t}$ is a Markov process on the half-sphere $B_{+}=\{v$ : $\left.|v|=U, e_{z} v \geq 0\right\}$ as follows: The times of tumbles $\left\{T_{i}: i \in \mathbf{N}\right\}$ is a renewal process, i.e. the durations of the runs $\left\{T_{i+1}-T_{i}: i \in \mathbf{N}\right\}$ are independent. After a tumble at time $t=T_{i}$, a new velocity $V_{t}$ is chosen uniformly from the half-sphere with respect to solid angle. Between tumbles, the velocity undergoes Brownian motion on the half-sphere with rotational diffusivity $\kappa$, i.e. $\mathbf{E}\left(\angle\left(V_{t}, V_{t+h}\right)\right)^{2}=2 \kappa h+o(h)$. The next tumble occurs when the velocity process hits the plane $e_{z} v=0$ so that the direction is perpendicular to the gradient, i.e. $\lim _{t \uparrow T_{i+1}} e_{z} V_{t}=0$.

The parameters in this model are the swimming speed $U$ [Length/Time] and the rotational diffusivity $\kappa$ [1/Time]. A simple dimensional analysis yields that the resulting chemotactic drift must scale with the swimming speed $U$ and be independent of the rotation $\kappa$, whereas the chemotactic dispersal scales with $U^{2} / \kappa$. The following result yields the coefficients in the scaling relations.

Theorem 3 For the model above we have the central limit theorem

$$
\frac{1}{\sqrt{t}}\left(X_{t}-v t\right) \rightarrow N(0,2 D) \text { in distribution as } t \rightarrow \infty
$$

where $v=U \cdot\left(0,0, v_{z}\right)$ with 


$$
v_{z} \rightarrow \frac{1}{8 \log 2-4} \approx 0.64
$$

while $D=U^{2} \kappa^{-1} \cdot \operatorname{diag}\left(D_{x}, D_{y}, D_{z}\right)$ with

$$
D_{z} \approx 1.00 \cdot 10^{-2}, \quad D_{x}=D_{y} \approx 8.54 \cdot 10^{-2}
$$

In stationarity, the expected time since last tumble is

$$
\lim _{t \rightarrow \infty} \mathbf{E} A_{t}=\kappa^{-1}\left(\frac{3-4 \log 2}{2 \log 2-1}+2 \log 2-1\right) \approx 0.975 \kappa^{-1}
$$

The proof is found in appendix D. Note, as predicted by the dimension analysis, that the chemotactic velocity is independent of the magnitude of the Brownian rotation. In particular, any non-zero Brownian rotation will decrease the maximum chemotactic velocity from the swimming speed $U$ to $0.64 U$.

The diffusivities can be compared with that of the same bacterium in absence of tumbling: There, the velocity decorrelates exponentially with a time constant of $1 / \kappa$; hence the diffusivity is isotropic and has magnitude $U^{2} /(3 \kappa)$. We see that the tumbles decrease the diffusivity by a factor of 4 , roughly, in the direction perpendicular to the gradient, and a factor of 33, roughly, in the direction of the gradient. The factor 4 is due to reduction of the decorrelation time, while the factor 33 is mostly due to reduction of the variance.

It is possible to generalize the preceding analysis to the case of finite chemotactic sensitivity $\beta$, although most of the resulting partial differential equations must be solved numerically.

Our original motivation for examining the model in this section was that it is the limit of the run-tumble model of section 3 when the parameter $\beta$ in 
(4) goes to infinity. Nevertheless, the model may have a relevance which does not rely on the functional form (4), since it simply states that the organism reorients itself when and only when it experiences a degrading environment. Even if this assumes a high fidelity sensory system and a crude behavioral strategy, it is a reasonable first model of tumbling search behavior.

\section{Discussion}

The contribution of this paper is, first, to demonstrate that Anscombe's theorem, a central limit theorem for renewal-reward processes, is useful for deriving diffusion limits of stochastic motion. Second, we followed this approach for the specific example of run-tumble motion with the standard exponential relationship between run direction and mean run length, including Brownian rotation. The main advantage of this approach, and consequently the value of Anscombe's theorem in this context, stems from conceptual and computational simplicity: Statistics of the single run may be obtained with well known and mathematically straightforward methods, after which the coarse-grained statistics of the motion may be obtained with elementary calculations using Anscombe's theorem. As an example of this, since no scale separation is used when analyzing the statistics of a single run, the nonlinearity and anisotropy of the response to chemical cues is preserved directly in the diffusion approximation.

The result, proposition 1, applies not only when the run statistics are obtained analytically as in section 3 , but also when they are determined by 
Monte Carlo as in the second part of theorem 3. In that case it suffices to simulate one single step repeatedly in order to estimate the run statistics. Even if run statistics are obtained with numerical analysis of partial differential equations, as also done in the second part of theorem 3 , these equations are standard and can be analyzed with well-known methods.

Similarly, the run statistics may be obtained empirically by observing motion of single individuals. If we have obtained maximum likelihood estimates of these statistics, we may insert these estimates in the formulas in the proposition to obtain maximum likelihood estimates of the chemotactic velocity and dispersal. In this situation, a problem with observed trajectories is that the division into runs may be partially subjective, e.g. through a threshold in local accelerations. In that case the threshold should be chosen so as to eliminate correlations between subsequent runs.

For the run-tumble motion, the dispersal turns out to be anisotropic, which is not surprising given that the motion is biased (Bearon, 2003; Codling et al, 2010). The diffusivity increases quite rapidly with the steepness of the gradient. This should be contrasted to the findings of Rivero et al (1989), who considered a similar one-dimensional model and showed that the dispersal is a decreasing function of gradient steepness. Our results demonstrate a fundamental difference between motion in one and three dimensions; results from onedimensional studies should not be extrapolated to three dimensions without scrutiny. Also, using different methods Bearon (2003) examined the diffusive limit from a tumble rate which depends on the angle between the swimming di- 
rection and the concentration gradient in a slightly different way than the one presented in this paper, and found that along-gradient diffusivity decreased with chemotactic strength. This demonstrates that differences in higher order term has qualitative implications for the along-gradient diffusivity. Since the diffusivity is the product of the variance on the velocity process and decorrelation time of the velocity process, and since chemotaxis decreases the variance but increases the decorrelation time, subtle differences can determine if the net effect of chemotaxis is increased or reduced diffusivity.

Related to this is an interesting observation, which may appear paradoxical: With the run/tumble model and exponential form of the tumbling rate, as studied in this paper, increasingly strong bias will cause the chemotactic drift to approach the swimming speed so that in the limit the bacterium is swimming straight up the gradient, but at the same time the diffusivities tend to infinity. The resolution of this seeming paradox is that the decorrelation time of the velocity process increases faster than the variance of the velocity decreases.

The results for the run-tumble model of chemotaxis rely on the specific functional relationship between the tumble rate and the experienced change in concentration, i.e. the standard model (2). Although this relationship is common in the literature, its validity in the strongly biased case, i.e. steep gradients or high chemotactic sensitivity, does not have overwhelming empirical support. It can therefore be argued that a detailed analysis of the model in the strongly biased case is less fertile, but nevertheless it is important to 
know the analytical behavior of standard model components. A related objection is that the model (2) ignores intracellular state dynamics (Xue, 2015) and in particular that these dynamics will not be at steady-state when the animal experiences steep gradients. This implies that our results address nonlinearities in the chemotactic response to steep gradients but not the delays and transients. This objection is valid and implies that our conclusions will have a smaller range of applicability than those resulting from a detailed study such as Xue (2015). Regardless, the simplicity and generality of the result in theorem 1 speaks for the applicability of the result, and combined with the standard model (2) our results, specifically theorem 2, provides a reference that more elaborate and detailed models can be compared against.

A technical limitation of the renewal approach is that subsequent runs must be stochastically independent for the central limit theorem to apply. In the context of bacterial chemotaxis, it is a common empirical observation that subsequent runs are not independent, but that the direction before and after a tumble are positively correlated (Berg, 1993). It should be possible to relax the requirement of independence in the current framework and allow dependent but exponentially mixing runs, for example using the methods in Nielsen et al (2007), which rely on Markov renewal processes. One would expect that the chemotactic drift can be computed from run statistics as in proposition 1, i.e. as $\mu / \tau$, under very weak assumptions on correlation between runs, even if the statistics $\mu, \tau$ may themselves depend on interrun dependency (Locsei, 
2007). Meanwhile, positive correlation between run displacements is expected to enhance dispersal (Berg, 1993).

Comparing techniques for obtaining diffusion approximations, an advantage of the singular perturbation expansion is that it is systematic in the sense that it yields a series expansion where in principle arbitrary many terms can be computed, although with rapidly increasing effort. In contrast, the central limit theorem yields an approximation but offers no simple remedies if the accuracy is insufficient. Neither method of obtaining diffusion approximations provide simple methods for quantifying the approximation error, except through numerical simulation of the microscopic model.

The accuracy of diffusion approximations is, in general, a difficult topic. Techniques which are based on the autocorrelation of the velocity may yield not only the asymptotically dominating term which is linear in time, but also dominated terms which typically decay exponentially (Sandoval et al, 2014); this addresses the issue of finite time, but not the issue of processes being not completely stationary in a heterogeneous environment. It is to be considered good practice to compare decorrelation scales, in time and space, with other characteristic scales of the problem and scales of interest in the study, as e.g. is done by Bearon (2007). For this purpose, the characteristic scales obtained in section 4 are useful. In the standard model of chemotaxis (1), (2), for the parameter $\beta$ to display sufficiently small variability over many runs the concentration must remain far below saturation and the gradient must be relatively constant; assumptions that may be violated in steep gradients. 
Furthermore, even if a diffusion process may approximate the run-tumble process, the standard run-tumble model may be an inadequate representation of the actual organism, for example because transients in intracellular dynamics become important (Xue, 2015). Detailed simulation studies are required to examine the range of applicability of each of these approximations.

Ultimately, whether a diffusion approximation is justified depends also on the question being asked. For example, for the case of bacteria with run/tumble behavior and infinite sensitivity as in section 5 , diffusion approximations may provide accurate statistics for the displacement of a bacterium over a given time interval. However, regarding the density of bacteria, a fundamental difference is that the microscopic model in this limiting case always predicts a flux up the gradient of the chemoattractant, whereas the flux in the diffusion approximation depends also on the gradient of the bacterial density, and therefore may go against the gradient of the chemoattractant in certain situations.

To conclude, we have provided a technique for deriving diffusion approximations, which assumes independent segments (runs) of the trajectories, and relates the drift and dispersal from the statistics of these runs. The usefulness of this technique stems from the simplicity of the relationship between run statistics and the resulting chemotactic drift and dispersal, which facilitates determining diffusion approximations either through mathematical analysis, Monte Carlo simulation, or statistical analysis of observed motion. Due to this simplicity, the technique is a valuable supplement to other existing methods for deriving diffusion approximations. 


\section{A Proof of proposition 1}

Define the scalar stochastic process

$$
z_{i}=c^{\prime} x_{i}-\frac{c^{\prime} \mu}{\tau} t_{i}
$$

where $c$ is an arbitrary column vector and $c^{\prime}$ denotes transpose. $z_{i}$ is a scalar auxillary process which has been constructed so as to have mean $\mathbf{E} z_{i}=0$, thus enabling us to apply Anscombe's theorem. Its variance is

$$
\mathbf{V} z_{i}=\sigma^{2}=c^{\prime}\left(\Sigma_{x}-\frac{1}{\tau}\left(\mu \rho^{\prime}+\rho \mu^{\prime}\right)+\frac{\mu \mu^{\prime}}{\tau^{2}} \sigma_{t}^{2}\right) c=c^{\prime} \Sigma c
$$

Define

$$
S_{n}=\sum_{i=1}^{n} z_{i}
$$

and note that by the law of large numbers, $t^{-1} N(t) \rightarrow \tau^{-1}$ almost surely. We may thus apply Anscombe's theorem to conclude that $S_{N(t)}$ is asymptotically normally distributed. Now, $S_{n}$ is closely related to the cumulated reward $\bar{X}_{T}$ which is our object of investigation:

$$
S_{N(t)}=c^{\prime} \bar{X}_{t}-\frac{c^{\prime} \mu}{\tau} T_{N(t)}=c^{\prime} \bar{X}_{t}-\frac{c^{\prime} \mu}{\tau} t+\frac{c^{\prime} \mu}{\tau} A_{t}
$$

where

$$
A_{t}=t-T_{N(t)}
$$

is the age, i.e. the time that has passed since since last tumble. The process $A_{t}$ is bounded in probability, and hence

$$
\frac{A_{t}}{\sqrt{t}} \rightarrow 0 \text { in probability as } t \rightarrow \infty
$$

Thus, the asymptotic distribution of the sum $S_{N(t)}$ implies a similar property of $\bar{X}_{t}$ :

$$
c^{\prime} \frac{\bar{X}_{t}-\frac{\mu}{\tau} t}{\sqrt{t / \tau}}=\frac{S_{N(t)}}{\sqrt{t / \tau}}-\frac{\mu}{\sqrt{\tau}} \frac{A_{t}}{\sqrt{t}} \rightarrow N(0, \sigma) \text { in distribution. }
$$

Since this holds for any $c, \bar{X}_{t}$ itself must be asymptotically normally distributed:

$$
\frac{1}{\sqrt{t}}\left(\bar{X}_{t}-\frac{\mu}{\tau} t\right) \rightarrow N(0, \Sigma / \tau) \text { in distribution. }
$$




\section{B Proof of theorem 2}

We aim to apply proposition 1 and therefore pursue the statistics of a single run. For simplicity we rescale time and length so that the swimming speed is 1 and the base tumble rate is 1 , and express the tumble rate as a function of the angle $\theta$ between $v$ and $e_{z}$. Then, the tumble rate is

$$
\lambda(\theta)=e^{-\beta \cos \theta}
$$

At each tumble a new run direction is chosen, uniformally with respect to solid angle. I.e., the angle $\theta_{i}$ between the $z$-axis and the velocity during run $i$ is distributed between 0 and $\pi$ with density:

$$
\phi(\theta)=\frac{1}{2} \sin \theta \quad \text { for } 0 \leq \theta \leq \pi .
$$

The duration $t_{i}$ of a run is, when conditioning on the angle $\theta_{i}$, exponentially distributed with mean $1 / \lambda\left(\theta_{i}\right)$. We then obtain the unconditional mean:

$$
\tau=\mathbf{E} t_{i}=\mathbf{E}\left(\mathbf{E}\left\{t_{i} \mid \theta_{i}\right\}\right)=\mathbf{E} \frac{1}{\lambda\left(\theta_{i}\right)}=\int_{0}^{\pi} \frac{\phi(\theta)}{\lambda(\theta)} d \theta=\frac{1}{2 \beta}\left(e^{\beta}-e^{-\beta}\right)=\frac{\sinh \beta}{\beta} .
$$

This mean duration is a time scale which characterizes the process. It is examined in section 4 and compared to other time scales, and graphed in figure 2 .

Likewise, conditional on the angle $\theta_{i}$, the second moment of the run duration is $\mathbf{E}\left\{t_{i}^{2} \mid \theta_{i}\right\}=$ $2 / \lambda^{2}\left(\theta_{i}\right)$. We thus find the unconditional second moment:

$$
\mathbf{E} t_{i}^{2}=\mathbf{E}\left(\mathbf{E}\left\{t_{i}^{2} \mid \theta_{i}\right\}\right)=\mathbf{E} \frac{2}{\lambda^{2}\left(\theta_{i}\right)}=\int_{0}^{\pi} \frac{2 \phi(\theta)}{\lambda^{2}(\theta)} d \theta=\frac{1}{2 \beta}\left(e^{2 \beta}-e^{-2 \beta}\right)=\frac{\sinh 2 \beta}{\beta}
$$

from which we obtain the variance of the run duration:

$$
\sigma_{t}^{2}=\mathbf{V} t_{i}=\mathbf{E} t_{i}^{2}-\left(\mathbf{E} t_{i}\right)^{2}=\frac{\sinh 2 \beta}{\beta}-\frac{\sinh ^{2} \beta}{\beta^{2}}
$$

We now turn to the statistics of the displacement during a run, which we - with a slight abuse of notation - represent with coordinates $\left(x_{i}, y_{i}, z_{i}\right)$. We have the geometric relationship $z_{i}=t_{i} \cdot \cos \theta_{i}$ which yields the mean displacement in the $z$-direction: 


$$
\mu_{z}=\mathbf{E} z_{i}=\mathbf{E}\left(\mathbf{E}\left\{z_{i} \mid \theta_{i}\right\}\right)=\mathbf{E} \frac{\cos \theta_{i}}{\lambda\left(\theta_{i}\right)}=\int_{0}^{\pi} \frac{\phi(\theta)}{\lambda(\theta)} \cos \theta d \theta=\frac{\cosh \beta}{\beta}-\frac{\sinh \beta}{\beta^{2}}
$$

Rotational symmetry yields $\mu_{x}=\mu_{y}=0$. Proposition 1 now gives the drift in the diffusion approximation:

$$
v_{x}=v_{y}=0, \quad v_{z}=\frac{\mu_{z}}{\tau}=\operatorname{coth} \beta-\frac{1}{\beta}
$$

as shown in figure 1 .

Next, we need the second order properties of the displacement during a run. Rotational symmetry and Pythagoras' theorem yields

$$
\mathbf{E} x_{i}^{2}=\mathbf{E} y_{i}^{2} \text { and } x_{i}^{2}+y_{i}^{2}=t_{i}^{2} \sin ^{2} \theta_{i}
$$

Combining these two, we obtain the variance of displacement in the direction of the $x$-axis (or the $y$-axis):

$$
\begin{aligned}
\mathbf{E} x_{i}^{2} & =\frac{1}{2} \mathbf{E}\left(\mathbf{E}\left\{\left(t_{i}^{2} \sin ^{2} \theta_{i}\right) \mid \theta_{i}\right\}\right) \\
& =\mathbf{E} \frac{\sin ^{2} \theta_{i}}{\lambda^{2}\left(\theta_{i}\right)} \\
& =\int_{0}^{\pi} \frac{\phi(\theta) \sin ^{2} \theta}{\lambda^{2}(\theta)} d \theta \\
& =\frac{\cosh 2 \beta}{2 \beta^{2}}-\frac{\sinh 2 \beta}{4 \beta^{3}}
\end{aligned}
$$

Correspondingly, for the $z$-direction:

$$
\begin{aligned}
\mathbf{E} z_{i}^{2} & =\mathbf{E}\left(\mathbf{E}\left\{t_{i}^{2} \cos ^{2} \theta_{i} \mid \theta_{i}\right\}\right) \\
& =\mathbf{E} \frac{2 \cos ^{2} \theta_{i}}{\lambda^{2}\left(\theta_{i}\right)} \\
& =\int_{0}^{\pi} \frac{2 \phi(\theta) \cos ^{2} \theta}{\lambda^{2}(\theta)} d \theta \\
& =\frac{2 \beta^{2}+1}{2 \beta^{3}} \sinh 2 \beta-\frac{\cosh 2 \beta}{\beta^{2}}
\end{aligned}
$$

which yields the variance 


$$
\mathbf{V} z_{i}=\mathbf{E} z_{i}^{2}-\left(\mathbf{E} z_{i}\right)^{2}=\frac{\left(4 \beta^{3}+6 \beta\right) \sinh 2 \beta-\left(6 \beta^{2}+2\right) \cosh 2 \beta+2-2 \beta^{2}}{4 \beta^{4}}
$$

Rotational symmetry dictates that the run displacement $\left(x_{i}, y_{i}, z_{i}\right)$ has diagonal covariance matrix $\Sigma_{x}=\operatorname{diag}\left(\mathbf{V} x_{i}, \mathbf{V} y_{i}, \mathbf{V} z_{i}\right)$ and that the covariance between run duration and displacement is 0 in the two directions $e_{x}$ and $e_{y}$, so

$$
\rho=\left(0,0, \boldsymbol{C o v}\left(z_{i}, t_{i}\right)\right)^{\prime}
$$

Here, the covariance between run duration $t_{i}$ and displacent $z_{i}$ in $z$-direction is obtained with similar calculations:

$$
\begin{aligned}
\mathbf{E} t_{i} z_{i} & =\mathbf{E}\left(\mathbf{E}\left\{t_{i} z_{i} \mid \theta_{i}\right\}\right) \\
& =\mathbf{E}\left(\mathbf{E}\left\{t_{i}^{2} \cos \theta_{i} \mid \theta_{i}\right\}\right) \\
& =\mathbf{E} \frac{2 \cos \theta_{i}}{\lambda^{2}\left(\theta_{i}\right)} \\
& =\int_{0}^{\pi} \frac{2 \phi(\theta) \cos \theta}{\lambda^{2}(\theta)} d \theta \\
& =\frac{\cosh \beta}{\beta}-\frac{\sinh 2 \beta}{2 \beta^{2}}
\end{aligned}
$$

yielding

$$
\operatorname{Cov}\left(t_{i}, z_{i}\right)=\mathbf{E} t_{i} z_{i}-\tau \mu_{z}=\frac{\left(2 \beta^{2}+1\right) \cosh 2 \beta-2 \beta \sinh 2 \beta-1}{2 \beta^{3}} .
$$

We now have the statistics which are required to apply proposition 1 . In the $x$ and $y$ direction we get

$$
D_{x}=D_{y}=\frac{\mathbf{E} x_{i}^{2}}{2 \tau}=\frac{2 \beta \cosh 2 \beta-\sinh 2 \beta}{8 \beta^{2} \sinh \beta}
$$

while in the $z$ direction we get

$$
D_{z}=\frac{\mathbf{V} z_{i}+\frac{\left(\mathbf{E} z_{i}\right)^{2}}{\tau^{2}} \mathbf{V} t_{i}-2 \frac{\mathbf{E} z_{i}}{\tau} \mathbf{C o v}\left(t_{i}, z_{i}\right)}{2 \tau}=\frac{1}{4} \frac{\sinh 2 \beta-2 \beta}{\beta^{2} \sinh \beta} .
$$

Proposition 1 now establishes the limiting Gaussian distribution of the position at tumbles, and it remains only to show that the same limiting distribution applies during a run: 
The displacement during a run from the position at last tumble will be bounded in probability. Therefore $\left(\bar{X}_{t}-X_{t}\right) / \sqrt{t}$ converges to 0 in probability, so that $\left(X_{t}-v t\right) / \sqrt{t}$ and $\left(\bar{X}_{t}-v t\right) / \sqrt{t}$ has the same limiting distribution, i.e. the limiting Gaussian distribution applies also to the position at any point in time, whether at a tumble or during a run.

\section{Monte Carlo simulation}

Here we describe the Monte Carlo simulation performed to verify the results of theorem 2; i.e. the estimates and associated confidence intervals displayed in figure 1. The run-tumble model with constant speed $U=1$, base tumble rate $\lambda_{0}=1$ and different values of the parameter $\beta$, is simulated stochastically. The initial distribution for the velocity is the stationary distribution which scales with $1 / \lambda(v)$; this is a truncated exponential distribution for $v_{z}=v \cdot e_{z}$ and conditional on this, a uniform distribution of the two other components on the circle $\left\{\left(v_{x}, v_{y}\right): v_{x}^{2}+v_{y}^{2}=1-v_{z}^{2}\right\}$. The motion is simulated one tumble at a time until the time of last tumble exceeds the simulation time $T=10000$; after this the position at time $T$ is obtained by interpolation in the last run and reported. This simulation time is orders of magnitude larger than the decorrelation time of the velocity process (compare figure 2) which ensures that the diffusion approximation is reasonable. $N=1000$ independent replicates of the motion are simulated for each value of $\beta$. Estimates of the drift and diffusivity are obtained as sample means computed from mean displacement and its variance, respectively. $95 \%$ confidence intervals for the mean are obtained using \pm 1.96 times the standard error between replicates; these intervals are smaller than the plotting symbols so practically invisible in the plot. $95 \%$ confidence intervals for the diffusivity are obtained from percentiles in $M=1,000$ bootstrap replicates.

\section{Proof of theorem 3}

We first non-dimensionalize the model; this amounts to taking $U=1$ and $\kappa=1$. We aim to apply proposition 1 and therefore examine the statistics of time and displacement during 
a single run. A run starts at time 0 with a random velocity $V_{0}$ sampled uniformly on the half-sphere $B_{+}$with respect to solid angle and ends when the Brownian motion on the half-sphere hits the plane $\left\{v: e_{z} v=0\right\}$.

We first transform the problem to a one-dimensional one by focusing on the velocity in the $z$-direction. During a run, the instantaneous velocity vector $V_{t}$ is Brownian motion on the surface of the unit sphere, which can be written as the solution to an Itô stochastic differential equation (Øksendal, 2010)

$$
d V_{t}=-V_{t} d t+\left(I-V_{t} V_{t}^{\prime}\right) d B_{t}
$$

where $B_{t}$ is three-dimensional Brownian motion. Let $U_{t}=e_{z} V_{t}$ denote the velocity in the direction of the gradient. Using Itô's lemma we obtain

$$
d U_{t}=-U_{t} d t+\sqrt{1-\left|U_{t}\right|^{2}} d W_{t}
$$

Here

$$
d W_{t}=\frac{1}{\sqrt{1-\left|U_{t}\right|^{2}}} e_{z}\left(I-V_{t} V_{t}^{\prime}\right) d B_{t}
$$

and, noting that

$$
\left|\frac{1}{\sqrt{1-\left|U_{t}\right|^{2}}} e_{z}\left(I-V_{t} V_{t}^{\prime}\right)\right|^{2}=1
$$

we recognize $W_{t}$ as standard Brownian motion (compare Øksendal (2010)). Thus the process is $U_{t}$ is itself a Markov process with generator $L$ given by

$$
(L f)(u)=-u \frac{\partial f}{\partial u}+\frac{1}{2}\left(1-u^{2}\right) \frac{\partial^{2} f}{\partial u^{2}}=\frac{\partial}{\partial u}\left(\frac{1}{2}\left(1-u^{2}\right) \frac{\partial f}{\partial u}\right)
$$

Up to the factor $1 / 2$, this is Legendre's operator of order 0 .

From the point of view of the individual run, the boundary $u=0$ is absorbing in that the run terminates when $U_{t}$ reaches this boundary. Let $T=\inf \left\{t: U_{t} \leq 0\right\}$ denote this stopping time. We seek the statistics of $T$ and $Z_{T}$. During the run, the time $t$ and upgradient displacement $Z_{t}$ are additive processes, studied in an oceanographic context by Thygesen et al (2007). Define the moment functions 


$$
\psi_{i j}(u)=\mathbf{E}^{u} T^{i} Z_{T}^{j}
$$

then it follows that these functions $\psi_{i j}$ for $i, j \in \mathbf{N}_{0},(i, j) \neq(0,0)$ are governed by boundary value problems

$$
\begin{aligned}
& L \psi_{10}+1=0 \\
& L \psi_{01}+u=0 \\
& L \psi_{i j}+i \psi_{(i-1) j}+j u \psi_{i(j-1)}=0 \text { for } i, j \in \mathbf{N}
\end{aligned}
$$

on the domain $u \in(0,1)$. The boundary condition at $u=0$ is $\psi_{i j}(0)=0$ while the

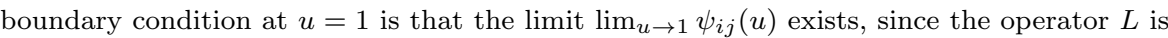
singular at that point (recall that $u=1$ does not correspond to a boundary point for the original Brownian motion on the half-sphere $B_{+}$).

For the low-order moments we find the solutions:

$$
\begin{aligned}
& \psi_{10}(u)=\mathbf{E}^{u} T=2 \log (1+u) \\
& \psi_{01}(u)=\mathbf{E}^{u} Z_{T}=u \\
& \psi_{20}(u)=\mathbf{E}^{u} T^{2}=8(\log 2-1) \log (u+1)-4(\log (2))^{2}-8 \operatorname{dilog}\left(\frac{u}{2}+\frac{1}{2}\right)+2 \frac{\pi^{2}}{3} \\
& \psi_{11}(u)=\mathbf{E}^{u} T Z_{T}=(4+2 z) \log (u+1)-2 u \\
& \psi_{02}(u)=\mathbf{E}^{u} Z_{T}^{2}=\frac{2}{3} u^{2}+\frac{4}{3} \log (u+1)
\end{aligned}
$$

Here the dilog function is $\operatorname{dilog}(x)=\int_{1}^{x}(1-t)^{-1} \log t d t$.

These statistics are conditional on the up-gradient velocity $u=U_{0}$ at the beginning of the run. To find the unconditional statistics, we take expectation with respect to the distribution of $U_{0}$. Since $V_{0}$ is uniformly distributed on the half-sphere $B_{+}$with respect to solid angle, $U_{0}$ is uniformly distributed on $[0,1]$. Hence

$$
\mathbf{E} T^{i} Z_{T}^{j}=\int_{0}^{1} \psi_{i j}(u) d u
$$


We find:

$$
\begin{aligned}
\mathbf{E} T & =4 \log 2-2 \approx 0.77 \\
\mathbf{E} Z_{T} & =\frac{1}{2} \\
\mathbf{E} T^{2} & =16(1-\log 2)^{2} \approx 1.51 \\
\mathbf{E} T Z_{T} & =-9 / 2+8 \log 2 \approx 1.05 \\
\mathbf{E} Z_{T}^{2} & =-\frac{10}{9}+\frac{8}{3} \log 2 \approx 0.74
\end{aligned}
$$

According to proposition 1, we can now compute the advective velocity as

$$
\frac{\mathbf{E} Z_{T}}{\mathbf{E} T}=\frac{1}{8 \log 2-4} \approx 0.64
$$

To determine the diffusivity, we need the variance of the run duration

$$
\mathbf{V} T=\mathbf{E} T^{2}-(\mathbf{E} T)^{2}=12-16 \log 2 \approx 0.91,
$$

the variance of the up-gradient displacement during a run

$$
\mathbf{V} Z_{T}=\mathbf{E} Z_{T}^{2}-\left(\mathbf{E} Z_{T}\right)^{2}=-\frac{49}{36}+\frac{8}{3} \log 2 \approx 0.49,
$$

and the covariance

$$
\operatorname{Cov}\left(T, Z_{T}\right)=\mathbf{E} T Z_{T}-\left(\mathbf{E} Z_{T}\right)(\mathbf{E} T)=-7 / 2+6 \log 2 \approx 0.66
$$

With this we can employ proposition 1 to obtain the diffusivity in the $z$-direction:

$$
\begin{aligned}
D_{z} & =\frac{1}{2} \frac{\mathbf{V} Z_{T}+\frac{\left(\mathbf{E} Z_{T}\right)^{2}}{(\mathbf{E} T)^{2}} \mathbf{V} T-2 \mathbf{E} Z_{T} \frac{\rho}{\mathbf{E} T}}{\mathbf{E} T} \\
& =\frac{1}{144} \frac{-85+490 \ln (2)-796(\ln (2))^{2}+384(\ln (2))^{3}}{(-1+2 \ln (2))^{3}} \\
& \approx 1.00 \cdot 10^{-2}
\end{aligned}
$$

By rotational symmetry, the chemotactic drift in the $x$ (or $y$ ) direction is zero, and the diffusivity matrix is diagonal. It remains to find the diffusivity $D_{x}$ in the $x$-direction, and 
to this end we must find $\mathbf{V} X_{T}$. Conditional on $V_{0}$, this is governed by the two boundary value problems on the half-sphere $B_{+}$:

$$
\nabla^{2} \gamma_{1}+x=0, \quad \nabla^{2} \gamma_{2}+2 x \gamma_{1}=0
$$

with Dirichlet boundary condition on the boundary $\left\{(x, y, z): z=0, x^{2}+y^{2}=1\right\}$. These equations do not appear to admit analytical solution in closed form. In stead, two numerical approaches were used: First, numerical solution of the two equations using the finite element software Comsol. Next, a Monte Carlo method where the stochastic differential equation (5) is simulated using a modified Euler method until absorption. The two numerical methods both estimate

$$
\mathbf{E} X_{T}^{2}=0.131 \pm 0.001
$$

With this, we find

$$
D_{x}=\frac{\mathbf{E} X_{T}^{2}}{2 \mathbf{E} T} \approx 8.54 \cdot 10^{-2}
$$

Finally, according to the general result (3), the expected age in stationarity is

$$
\mathbf{E} A_{t}=\frac{1}{2} \frac{\mathbf{V} T}{\mathbf{E} T}+\frac{1}{2} \mathbf{E} T=\frac{3-4 \log 2}{2 \log 2-1}+2 \log 2-1 \approx 0.975 .
$$

\section{References}

Adler J (1966) Chemotaxis in bacteria. Science 153(3737):708-716

Alt W (1980) Biased random walk models for chemotaxis and related diffusion approximations. J Math Biol 9:147-177

Anscombe F (1952) Large-sample theory of sequential estimation. Proc Cambridge Philos Soc 48:600-607

Bearon R (2001) Run-and-tumble chemotaxis in an ambient fluid flow. PhD thesis, University of Cambridge 
Bearon R (2003) An extension of generalized taylor dispersion in unbounded homogeneous shear flows to run-and-tumble chemotactic bacteria. Physics of Fluids 15:1552

Bearon R (2007) A model for bacterial colonization of sinking aggregates. Bulletin of Mathematical Biology 69(1):417-431

Bearon R, Pedley T (2000) Modelling run-and-tumble chemotaxis in a shear flow. Bulletin of Mathematical Biology 62:775-791

Benhamou S (2014) Of scales and stationarity in animal movements. Ecology Letters 17(3):261-272, DOI 10.1111/ele.12225, URL http://dx.doi.org/10.1111/ele.12225

Berg H (1993) Random Walks in Biology, 2nd edn. Princeton University Press

Brown D, Berg H (1974) Temporal stimulation of chemotaxis in Escherichia coli. Proc Nat Acad Sci 71(4):1388-1392

Cluzel P, Surette M, Leibler S (2000) An ultrasensitive bacterial motor revealed by monitoring signaling proteins in single cells. Science 287(5458):1652-1655

Codling E, Bearon R, Thorn G (2010) Diffusion about the mean drift location in a biased random walk. Ecology 91(10):3106-3113

Colin R, Zhang R, Wilson L (2014) Fast, high-throughput measurement of collective behaviour in a bacterial population. Journal of The Royal Society Interface 11(98):20140,486

Frankel I, Brenner H (1989) On the foundations of generalized taylor dispersion theory. Journal of Fluid Mechanics 204(-1):97-119

Grimmett G, Stirzaker D (1992) Probability and Random Processes, 2nd edn. Oxford University Press

Grünbaum D (1999) Advection-diffusion equations for generalized tactic searching behaviors. J Math Biol 38:169-194

Grünbaum D (2000) Advection-diffusion equations for internal state-mediated random walks. SIAM J Appl Math 61(1):43-73

Hastings A, Petrovskii S, Morozov A (2011) Spatial ecology across scales. Biology Letters 7(2):163-165, DOI 10.1098/rsbl.2010.0948

Jackson G (1987) Simulating chemosensory response of marine microorganisms. Limnol Oceanogr 32:1253-1266 
Jackson G (1989) Simulation of bacterial attraction and adhesion to falling particles in an aquatic environment. Limnol Oceanogr 34(3):514-530

Kay R, Langridge P, Traynor D, Hoeller O (2008) Changing directions in the study of chemotaxis. Nature Reviews Molecular Cell Biology 9(6):455-463

Keller E, Segel L (1971) Model for chemotaxis. J theor Biol 30:225-234

Kiørboe T, Jackson G (2001) Marine snow, organic solute plumes, and optimal chemosensory behavior of bacteria. Limnol Oceanogr 46(6):1309-1318

Kiørboe T, Ploug H, Thygesen UH (2001) Fluid motion and solute distribution around sinking aggregates. I. Small scale fluxes and heterogeneity of nutrients in the pelagic environment. Marine Ecology Progress Series 211:1-13

Locsei J (2007) Persistence of direction increases the drift velocity of run and tumble chemotaxis. Journal of Mathematical Biology 55(1):41-60

Nielsen BF, Nilsson LF, Thygesen UH, Beyer JE (2007) Higher order moments and conditional asymptotics of the batch markovian arrival process. Stochastic models 23(1):1-26

Øksendal B (2010) Stochastic Differential Equations - An Introduction with Applications, sixth edn. Springer-Verlag

Okubo A, Levin S (2001) Diffusion and Ecological Problems: Modern Perspectives. Springer

Othmer H, Hillen T (2002) The diffusion limit of transport equations II: Chemotaxis equations. SIAM J Appl Math 62(4):1222-1250

Othmer H, Dunbar S, Alt W (1988) Models of dispersal in biological systems. J Math Biol $26: 263-298$

Patlak C (1953) Random walk with persistence and external bias. Bulletin of Mathematical Biophysics 15:311-338

Rivero M, Tranquillo R, Buettner H, Lauffenburger D (1989) Transport models for chemotactic cell populations based on individual cell behavior. Chem Eng Sci 44(12):2881-2897 Sandoval M, Marath NK, Subramanian G, Lauga E (2014) Stochastic dynamics of active swimmers in linear flows. Journal of Fluid Mechanics 742:50-70

Taylor G (1921) Diffusion by continuous movements. Proc Lon Math Soc 20:196-211 
Thygesen U, Nilsson A, Andersen K (2007) Eulerian techniques for individualbased models based on additive processes. J Marine Systems 67:179-188, DOI 10.1016/j.marsys.2006.10.005

Xue C (2015) Macroscopic equations for bacterial chemotaxis: integration of detailed biochemistry of cell signaling. Journal of mathematical biology 70(1-2):1-44 